\title{
Study on Installation Angle of the Milling Wheel Accurate Estimation and Compensation
}

\author{
ZongXuemei ${ }^{1}$, Guo Qiang ${ }^{2}$, Kang Kaixuan ${ }^{1}$, JiaHaibo $^{1}$ and He bing ${ }^{1}$ \\ ${ }^{1}$ Jiangsu Xuzhou Construction Machinery Research Institute, Xuzhou 221004, Jiangsu, China \\ ${ }^{2}$ School of Mechanical and Power Engineering, Henan Polytechnic University, Jiaozuo, 454000, China
}

\begin{abstract}
An accurate estimation and compensation algorithm is proposed to calculate the manufacture deviation of the milling wheel based on optical measurement. Firstly, the spatial position of the cutting tool tip and the installation angle of the cutting tool are calculated based on measured data from hand held coordinate measuring instrument. Secondly, the installation angle field between the measurement model and CAD modelis calculated and analyzed by statistics methods. Thirdly, the deviation compensation strategy is given to correct the manufacturing process base on the results of the analysis. The feasibility of the proposed method has been validated by an illustrated experiment at last. The comparison results show that the deviation of the installation angle can be effectively constrained and the proposed method is helpful for the improvement of quality and accuracy in milling wheel.
\end{abstract}

\section{Introduce}

As the key part of the milling machine, the milling wheel plays an important role in the working performance. The road material is cut down in turn by the cutting tool, and concentrated in the middle of the wheel by the spiral blades which are arranged in the milling wheel. The road material is transferred into belt conveyor by cast material plate. The quality of the milling wheel greatly affects the service life and construction effect of the milling machine. During the manufacture, whether or not the installation angle reaches the designed accuracy is a problem $[1,2]$. The tool apron is fixed on the milling drum by welding. Because of the deviation of the welding fixture, welding heat deformation, welding surrounding and welding process, there is a certain installation angle deviation between the design and manufacture.

With the high speed development of the optical technology, the optical measurement technology is widely used to measure the geometric deviation $[1,2]$. The traditional method to measure the welding heat deformation is mainly applied to regulation components and parts $[3,4]$. The optical measurement that is applied to irregular components and parts is rare. For that reason, an accurate estimation and compensation algorithm is proposed to calculate the manufacture deviation of the milling wheel based on optical measurement in this paper. Firstly, the spatial position of the cutting tool tip and the installation angle of the cutting tool are calculated based on measured data from handheld coordinate measuring instrument. Secondly, the installation angle field between the measurement model and CAD model is calculated and analyzed by statistics methods. Thirdly, the deviation compensation strategy is given to correct the manufacturing process base on the results of the analysis. The method has realized the accurate estimation of each tool deformation of the milling wheel, and it can offer correct guidance for the amendment of the design and manufacture.

\section{Installation angle analysis}

A reasonable installation angle has important implication for the service life and construction effect and surrounding. An unreasonable installation angle may lead to abnormal wear of the cutting tool, vibration of the machine and safety of the workers $[5,6]$.

The spatial position of the cutting tool is determined jointly by the spatial position of the cutting tool tip and the installation angle of the cutting tool. As shown in the Fig.1, Cartesian coordinate system $\mathbf{O X Y Z}$ set on the end surface of the milling drum. The rotational axis of the milling drum is represented by a vector $\boldsymbol{Z}(u)$. The cutting tool coordinate is defined as the part coordinate system $\mathbf{O}_{1} \mathbf{X}_{1} \mathbf{Y}_{1} \mathbf{Z}_{1}$ that is obtained by translating the $\mathbf{O X Y Z}$ a distance along the $\mathbf{Z}$ axis and a radius of the cutting tool tip circle along the $\mathbf{X}$ axis and rotating the $\mathbf{O X Y Z}$ a circumferential angle $\theta$ around the $\mathbf{Z}$ axis.

The installation angle consists of the cutting angle $\varphi$ and roll angle $\delta$, as show in the Fig.1, the cutting angle $\varphi$ is defined as the included angle between the rotational axis $\boldsymbol{H}(u)$ of the cutting tool and the center line $\boldsymbol{M}(u)$ of the cutting tip circle, which is the axis $\mathbf{X}_{1}$ in the part coordinate system. The roll angle $\delta$ is defined as the 
included angle between the rotational axis of the cutting tool and the forward direction of the cutting tool, and is also described as the included angle between the end face OXY of the milling drum and the roll face $\boldsymbol{P}(u)$ which is determined by the rotational axis $\boldsymbol{H}(u)$ of the cutting tool and the axis $\mathbf{X}_{\mathbf{1}}$.

Define:

- $\alpha, \beta$ and $\gamma$ are the included angle between the rotational axis of the cutting tool and axis $\mathrm{X}, \mathrm{Y}$ and $\mathrm{Z}$ in coordinate system OXYZ, respectively.

- $\alpha_{1}, \beta_{1}$ and $\gamma_{1}$ are the included angle between the rotational axis of the cutting tool and axis $\mathrm{X}_{1}, \mathrm{Y}_{1}$ and $\mathrm{Z}_{1}$ in the part coordinate system $\mathbf{O}_{1} \mathbf{X}_{1} \mathbf{Y}_{1} \mathbf{Z}_{1}$,respectively.

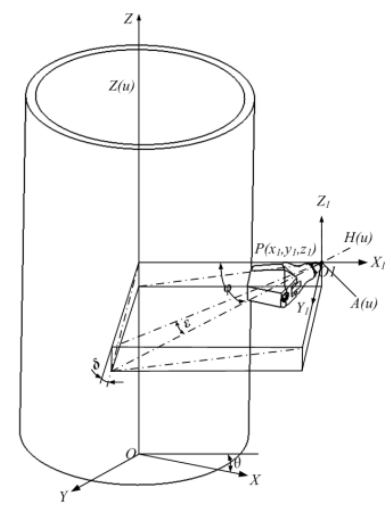

Figure 1. The installation angle model

As shown in the Fig. 1 , the $\alpha_{1}, \beta_{1}$ and $\gamma_{1}$ can be given as follows:

$$
\left\{\begin{array}{l}
\alpha_{1}=\varphi \\
\cos \beta_{1}=\cos \delta \cdot \sin \varphi \\
\cos ^{2} \alpha_{1}+\cos ^{2} \beta_{1}+\cos ^{2} \gamma_{1}=1
\end{array}\right.
$$

Then Eq. (1) can be simplified as

$$
\left\{\begin{array}{l}
\alpha_{1}=\varphi \\
\beta_{1}=\operatorname{arc}\lceil\cos \delta \cdot \sin \varphi\rceil \\
\gamma_{1}=\operatorname{arc}\lceil\sin \varphi \cdot \sin \delta\rceil
\end{array}\right.
$$

Variable $\varepsilon$ is represented the included angle between the projection of the rotational axis of the cutting tool in $\mathbf{O}_{1} \mathbf{X}_{1} \mathbf{Y}_{1}$ surface and the axis $\mathbf{X}_{1}$. Thus, the $\left(90^{\circ}{ }_{-\varepsilon}\right)$ is represented the included angle between the projection of the rotational axis of the cutting tool in $\mathbf{O}_{1} \mathbf{X}_{1} \mathbf{Y}_{1}$ surface and the axis $\mathbf{Y}_{\mathbf{1}}$.

$$
\left\{\begin{array}{l}
\alpha_{1}=\varphi \\
\cos \beta_{1}=\cos \delta \cdot \sin \varphi \\
\cos ^{2} \alpha_{1}+\cos ^{2} \beta_{1}+\cos ^{2} \gamma_{1}=1
\end{array}\right.
$$

According to the geometry model, the $\alpha_{l}, \beta_{l}$ and $\gamma_{1}$ can also be given as follows:

$$
\left\{\begin{array}{l}
\cos \alpha_{1}=\cos \varepsilon \cdot \cos \left(90^{\circ}-\gamma_{1}\right) \\
\cos \beta_{1}=\cos \left(90^{\circ}-\varepsilon\right) \cdot \cos \left(90^{\circ}-\gamma_{1}\right)
\end{array}\right.
$$

Then the variable $\varepsilon$ is calculated as

$$
\varepsilon=\arccos \left(\frac{\cos \alpha_{1}}{\sin \gamma_{1}}\right)
$$

Base on the same principle, $\varepsilon-\theta$ and $90^{\circ}-(\varepsilon-\theta)$ are represented the included angle between the projection of the rotational axis of the cutting tool in OXY surface and the axis $\mathbf{X}$ and $\mathbf{Y}$. The relations between $\alpha, \beta$ and $\gamma$ and the variable $\varepsilon$ can be given as

$$
\left\{\begin{array}{l}
\cos \alpha=\cos (\varepsilon-\theta) \cdot \cos \left(90^{\circ}-\gamma_{1}\right) \\
\cos \beta=\cos \left(90^{\circ}-\varepsilon+\theta\right) \cdot \cos \left(90^{\circ}-\gamma_{1}\right) \\
\gamma=\gamma_{1}
\end{array}\right.
$$

Furthermore, the rotational axis of the cutting tool can be given as

$$
\frac{\mathrm{X}-x_{1}}{\cos \alpha}=\frac{\mathrm{Y}-y_{1}}{\cos \beta}=\frac{\mathrm{Z}-z_{1}}{\cos \gamma}
$$

From Eq. (6), it can be seen that the rotational axis of the cutting tool has relationship with the six variables $(X$, $\mathrm{Y}, \mathrm{Z}, \alpha, \beta, \gamma)$. However, from Eq. (2) to Eq. (5), it can be seen that $\alpha, \beta$ and $\gamma$ can be represented byvariable $\varphi$ and $\delta$. Thus, the rotational axis of the cutting tool can be determined by $(\mathrm{X}, \mathrm{Y}, \mathrm{Z}, \varphi, \delta)$.

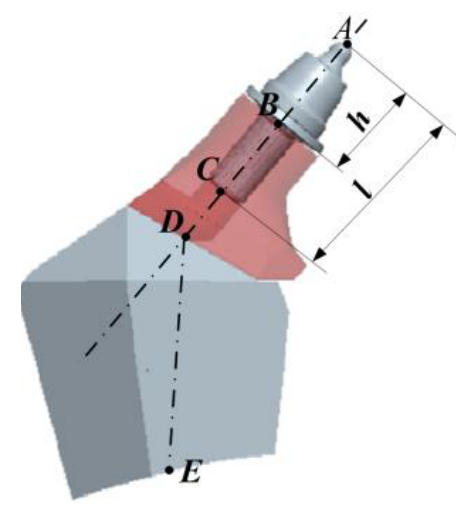

Figure 2. The tool apron model

If $l$ represents the length of the cutting tool, then the spatial position of the cutting tool root, as shown in Fig. 2, is obtained as

$$
\left(x_{1}-l \cos \alpha, y_{1}-l \cos \beta, z_{1}-l \cos \gamma\right)
$$

\section{Deviation measurement estimation}

\subsection{Elementary parameter measurement}

The elementary parameter of the milling wheel is measured by handheld coordinate measuring instrument that is based on laser triangulation method in this paper. As is shown in Fig.3, a laser, which is sent by the handheld coordinate measuring instrument, irradiate to the surface of an object to be measured, then an imaging will present on the double CCD sensor through reflection. With the displacement change of the surface of the object to be measured, the position of the imaging change. The position of the object to be measured is calculated through geometry model at last. 


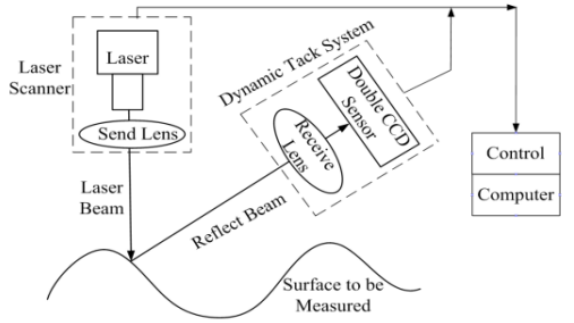

Figure 3. Laser triangulation method

As is shown in Fig.4, the rotational axis $\boldsymbol{Z}(\boldsymbol{u})$ of the milling drum and the end surface XOY of the milling drum are measured by reference to the original design firstly. The coordinate system OXYZ is built according to the measured data. Then location parameter that is consist of side face of the tool apron, end face and internal face of the cutting tool installation hole is obtained in the coordinate system OXYZ. The center point $\boldsymbol{C}(u)$ of the cutting tool installation hole and the rotational axis $\boldsymbol{H}(u)$ of the cutting tool are calculated through the measuring software.

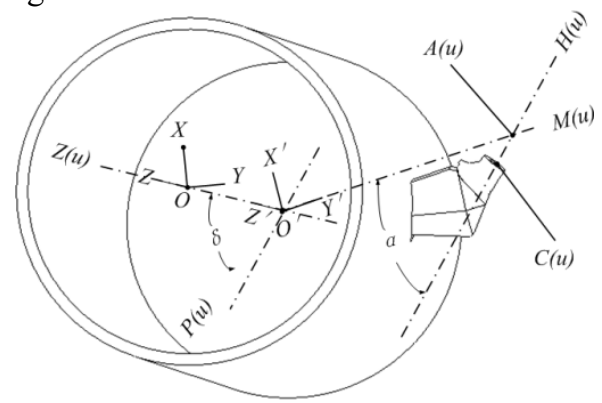

Figure 4. The measurement scheme

\subsection{Installation angle solution}

Based on the transformational relation of the elementary parameter and the installation angle, the transformational algorithm is built to calculate practical installation angle of the milling wheel. The detailed algorithm is shown as follows:

As is shown in Fig.4, the cutting tool tip $A(u)$ and the center point $\boldsymbol{C}(\mathrm{u})$ of the cutting tool installation hole are fall on the rotational axis $\boldsymbol{H}(u)$ of the cutting tool, thus the cutting tool tip $\boldsymbol{A}(u)$ can be written as,

$$
A(u)=C(u)+|h| H(u)
$$

Where $|h|$ represents the length of the cutting tool bit.

The cutting angle $\varphi$ is the included angle between the rotational axis $\boldsymbol{H}(u)$ of the cutting tool and the center line $\boldsymbol{M}(u)$ of the cutting tip circle, the Eq. (9) is given as

$$
\left\{\begin{array}{l}
M(u)=A(u)-O(u) \\
H(u) \cdot M(u)=|M(u)||H(u)| \cdot \cos \varphi
\end{array}\right.
$$

Where $\boldsymbol{O}(u)$ represents the gyration center of the cutting tool tip $A(u)$, then the cutting angle $\varphi$ is obtained as

$$
\varphi=\cos ^{-1} \frac{H(u) \cdot(A(u)-O(u))}{|H(u)||A(u)-O(u)|}
$$

The roll angle $\delta$ is the included angle between the end face $\boldsymbol{Z}(u)$ of the milling drum and the roll face $\boldsymbol{P}(u)$, the Eq. (9) is given as

$$
\left\{\begin{array}{c}
M(u)=A(u)-O(u) \\
P(u)=H(u) \times M(u) \\
Z(u) \cdot P(u)=|Z(u)||P(u)| \cdot \cos \delta
\end{array}\right.
$$

Then the roll angle $\delta$ is obtained as

$$
\delta=\cos ^{-1} \frac{Z(u) \cdot(H(u) \times(A(u)-O(u)))}{|H(u) \times(A(u)-O(u))|}
$$

\subsection{Deviation analysis and compensation}

Through the comparison for the original date and the practical date, the deviation of the installation angle is solved. The normality, the mean value and variance of the cutting angle and roll angle are calculated through Minitab software. The distribution of the installation angle field is analyzed.

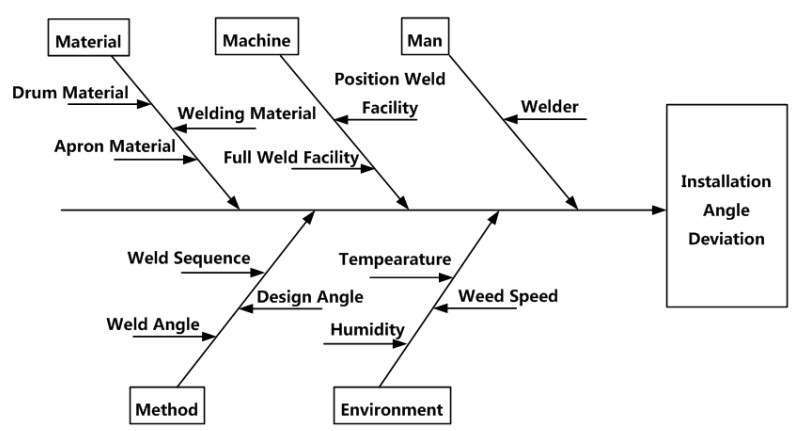

Figure 5. Fishbone diagram

Combined to the manufacture process of the milling wheel, the fishbone diagram method, as shown in Fig.5, is proposed to analyze the reason of the deviation from five respects, such as man, machine, materials, method and environment.

It can be found that the mean value deviation because of the welding deformation of the tool apron at the full weld stage, the main reason of the variance deviation is that the initial point and direction is inconsistent.

As you saw earlier, the rotational axis of the cutting tool can be determined by $(\mathrm{X}, \mathrm{Y}, \mathrm{Z}, \varphi, \delta)$. The $(\mathrm{X}, \mathrm{Y}, \mathrm{Z})$ is the spatial position of any point of the cutting tool axis. As is shown in Fig.2, the relative position of the five points (A B C D E) is fixed. Combined to the manufacture process of the milling wheel, the welding deformation, which is very small, can be neglected at the position welding stage. At the full weld stage, the large welding deformation of the tool apron causes the deviation of installation angle. However, the position of the center point of the tool apron at the bottom is still changeless. Therefore, it can take the angle reversible deformation method to compensate the mean value deviation of installation angle.

Meanwhile, improving the process capability of the manufacture and ensuring the consistent of the initial point and direction is important. It can reduce the 
variance deviation in order to guarantee the quality of the milling wheel.

\section{Experimental verification}

In the section, an illustrated experiment is given to evaluate the feasibility and applicability of the proposed measurement and compensation algorithm. The CAD model and the finished product of the same milling wheel, which are shown in Fig.6 and Fig.7, are used to validate the algorithm. About 88 tool aprons are set on the milling wheel regularly.

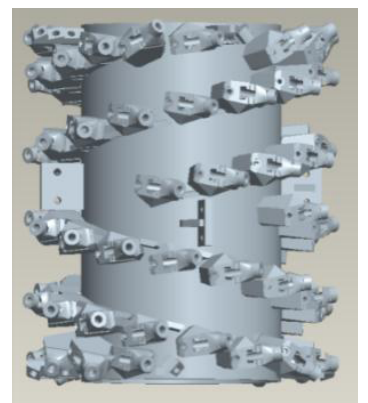

Figure 6. The $\mathrm{CAD}$ model of the milling wheel

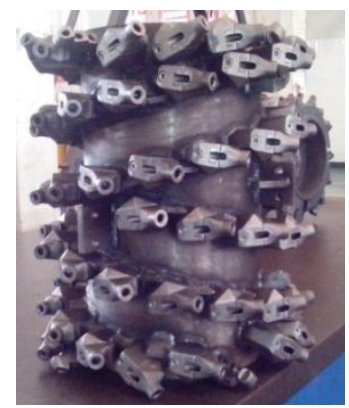

Figure 7. The finished product of the milling wheel

The comparison installation angle results of the CAD model and the finished product are given in Fig. 8 and Fig.9. From Fig.8, one can see that the mean value and variance of the cutting angle both exceed the bounding scope. The mean value is designed as 40 degree, while the mean value of manufacture is about 39.38 degree. Meanwhile, the value of the $C p$, which is represented the process capability of the manufacture, is only 0.87 . It is less than the standard value 1.33 .

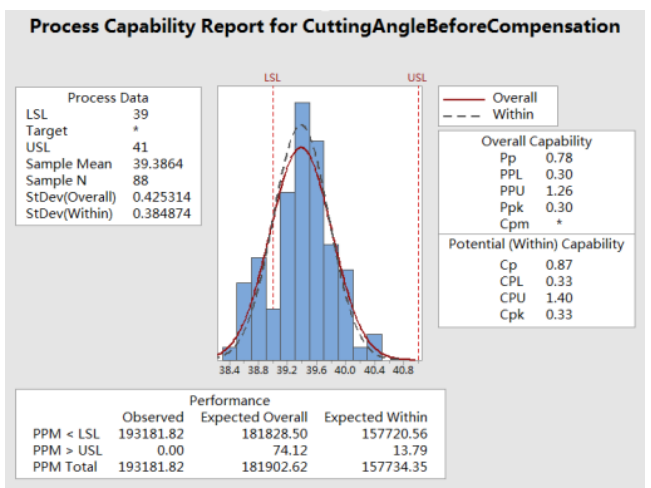

Figure 8. Process Capability Report for Cutting Angle Before

\section{Compensation}

From figure 9, it can be seen that the mean value of the roll angle, which is 7.46 degree, meet the design requirement which is designed as 40 degree. But the value of the $C p$ is just 0.63 . Therefore, it has a strong impact on the product quality.

The installation angle result of milling wheel that is optimized is shown in Fig.10 and Fig.11. It can be seen that the mean value and variance of the cutting angle both meet the design requirement.

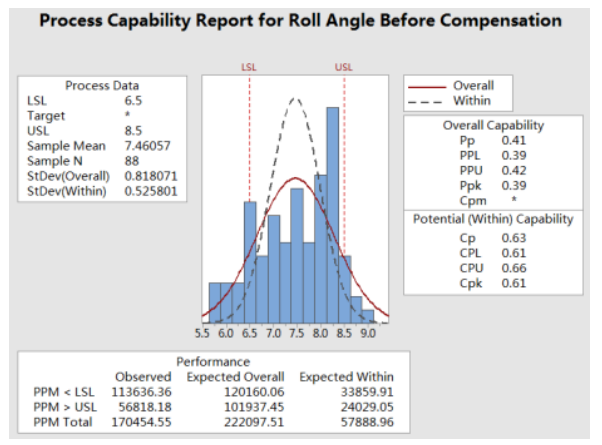

Figure 9. Process capability Report for roll angle before compensation

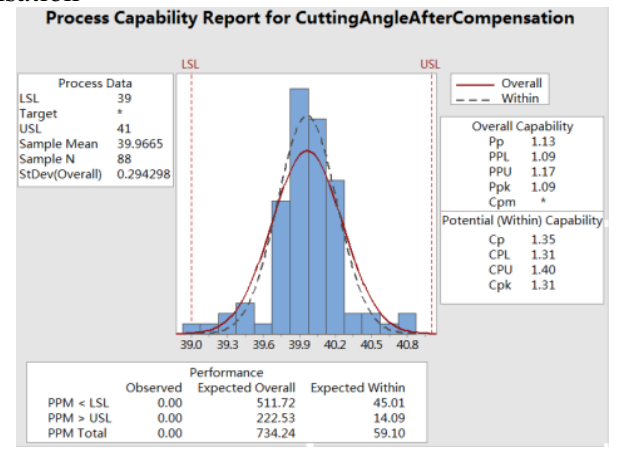

Figure 10. Process Capability Report for Cutting Angle After Compensation

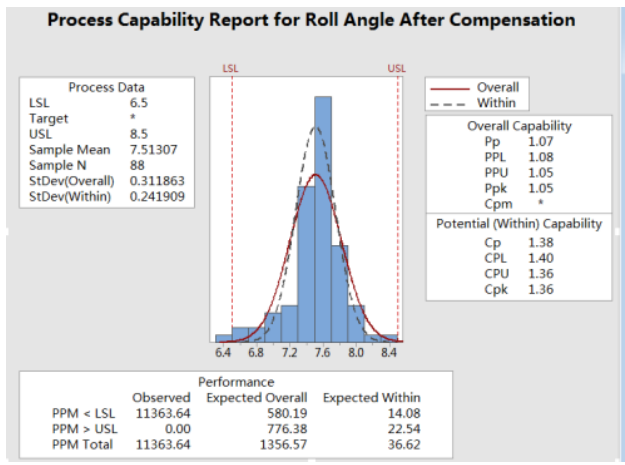

Figure 11. Process Capability Report for Roll Angle After Compensation

\section{Conclusions}

In this study, an accurate estimation and compensation algorithm was proposed to calculate the manufacture deviation of the milling wheel based on optical measurement. The scheduling method mainly concentrates on the assurance of manufacture accuracy and working performance of the milling wheel. Firstly, 
the elementary parameter of the milling wheel is measured by handheld coordinate measuring instrument. Then, the practical installation angle of the milling wheel is calculated and the process capability of the manufacture is analyzed. Furthermore, combine to the manufacture process of the milling wheel, the reversible deformation and process capability control method is proposed to compensate the deviation. The feasibility of the proposed method has been validated by an illustrated experiment. The comparison results show that the deviation of the installation angle can be effectively constrained and the proposed is helpful for the improvement of quality and accuracy in milling wheel.

\section{Acknowledgement}

This research is supported by Special Research Found for the Doctoral Program of Higher Education (Num. 20114116110002), Doctoral Fund of Henan Polytechnic University (Num. B2014-034), Foundation of He'nan Education Committee (No. 15A460025)

\section{References}

1. Jiang S.R., Zhang D.H., Wang W.H., Bu K., Cheng Y.W., Estimation of Displacement Field for Turbine Blade Profile Based on Reverse Engineering in Investment Casting. Special Casting \& Nonferrous Alloys, 29(1) (2009):13-15.

2. Gong Z.H., Zhong Z.H., Estimation of the Springback of Auto-Body PanelsBased on Reverse Engineering. Journal of Hunan University (Natural Sciences), 34(1) (2007):24-27, 55.

3. He H.Q., Zhao H.Y., Niu W.C., Wang P., A method to measure welding deformation of plate by three dimensional laser scanner. Transactions of the china welding institution, 32(12)(2011):9-12

4. Li L., Study on the rapid three-dimension optical measurement for welding deformation of sheet structure .Optical Technique, 36 (6)(2011):825- 828.

5. Tian J.Y., Xiang H.R., Analysis on parameter influence laws and milling resistances of pavement miller. Journal of Jiangsu University (Natural Science Edition), 25(5)(2004):380-384.

6. Gu H.R., Jiao S.J., Xiao C.Y., Analysis and Test of Milling Load Characteristics in Milling Machine. Chinese Journal of Highway and Transport, 25(3)(2012):154-156. 